in vivo $35: 3623-3626(2021)$

doi:10.21873/invivo.12668

\title{
A Rare Thymic Tumor - Lipofibroadenoma - Always a Postoperative Surprise
}

\author{
CIPRIAN BOLCA ${ }^{1,2,3}$, ALEXANDRU HAS $^{1,4}$, ANDREI BOBOCEA $^{1,5}$, ADELINA AFETELOR $^{1}$, RADU STOICA $^{6}$, \\ IRINA STRAMBU ${ }^{5,7}$, ILEANA STOICESCU ${ }^{7}$, ELENA MAGHERAN $^{8}$, IRINA BALESCU $^{9}$, \\ NICOLAE BACALBASA ${ }^{10,11}$, FLORENTINA GHERGHICEANU ${ }^{12}$, FLORENTINA FURTUNESCU ${ }^{13}$,

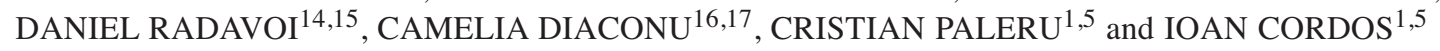 \\ ${ }^{1}$ Thoracic Surgery Department, "Marius Nasta” National Institute of Pneumology, Bucharest, Romania; \\ ${ }^{2}$ Faculty of Medicine and Health Sciences, Sherbrooke University, Sherbrooke, QC, Canada; \\ ${ }^{3}$ Thoracic Surgery Department, "Charles LeMoyne” Hospital, Longueuil, QC, Canada; \\ ${ }^{4}$ Thoracic Surgery Department, Kantonsspital St. Gallen, St. Gallen, Switzerland; \\ ${ }^{5}$ Thoracic Surgery Department, "Carol Davila” University of Medicine and Pharmacy, Bucharest, Romania; \\ ${ }^{6}$ Intensive Care Unit, "Marius Nasta” National Institute of Pneumology, Bucharest, Romania; \\ ${ }^{7} 5^{\text {th }}$ Pneumology Department, "Marius Nasta” National Institute of Pneumology, Bucharest, Romania; \\ ${ }^{8}$ Pathology Department, “Marius Nasta” National Institute of Pneumology, Bucharest, Romania; \\ ${ }^{9}$ Department of Surgery, "Ponderas” Academic Hospital, Bucharest, Romania; \\ ${ }^{10}$ Department of Obstetrics and Gynecology, "Carol Davila" \\ University of Medicine and Pharmacy, Bucharest, Romania; \\ ${ }^{11}$ Department of Visceral Surgery, Center of Excellence in Translational Medicine \\ "Fundeni" Clinical Institute, Bucharest, Romania; \\ ${ }^{12}$ Department of Marketing and Medical Technology, "Carol Davila" \\ University of Medicine and Pharmacy, Bucharest, Romania; \\ ${ }^{13}$ Department of Public Health and Management, University of \\ Medicine and Pharmacy "Carol Davila”, Bucharest, Romania; \\ ${ }^{14}$ Department of Urology, "Prof. Dr. Th. Burghele" Clinical Hospital, Bucharest, Romania; \\ ${ }^{15}$ Department of Urology, "Carol Davila” University of Medicine and Pharmacy, Bucharest, Romania; \\ ${ }^{16}$ Department of Internal Medicine, "Carol Davila” University of Medicine and Pharmacy, Bucharest, Romania; \\ ${ }^{17}$ Department of Internal Medicine. Clinical Emergency Hospital, Bucharest, Romania
}

\begin{abstract}
Background/Aim: Thymic lipofibroadenoma is a benign growth of unknown etiology extremely rarely described in the literature, morphologically resembling lipofibroadenoma of the breast. The diagnosis is usually a postoperative surprise and is made by the anatomopathologist. Surgical resection is curative. Case Report: The presented case is the first reported in a woman, to date. The patient was successfully submitted to surgery and complete resection of the giant intrathoracic tumor
\end{abstract}

This article is freely accessible online.

Correspondence to: Nicolae Bacalbasa, "Carol Davila" University of Medicine and Pharmacy, 37 Dionisie Lupu Street, 020021 Bucharest, Romania. Tel: +40 723540426, e-mail: nicolae_bacalbasa@yahoo.ro

Key Words: Thymic lipofibroadenoma, thymic rare tumor, resection. was performed. The histopathological and immunohistochemical studies came to demonstrated the presence of a thymic lipofibroadenoma while the postoperative outcomes were favorable. Conclusion: In cases presenting large intrathoracic tumors complete resection is able to provide a significant improvement of the general condition of the patient, as well as a chance for cure.

Thymic tumors are a rare disease compared to other types of intrathoracic malignancies being reported with an incidence of 1.5 per million $(1,2)$. The most commonly encountered thymic tumors are represented by thymomas and thymic carcinomas; except these categories, another subgroup, generally known as "thymic tumors of rare histology" has been described. These tumors are usually found randomly by the anatomopathologist, after surgical resection. Information concerning most of them is available in the literature exclusively as case reports, same as the one presented herein. 


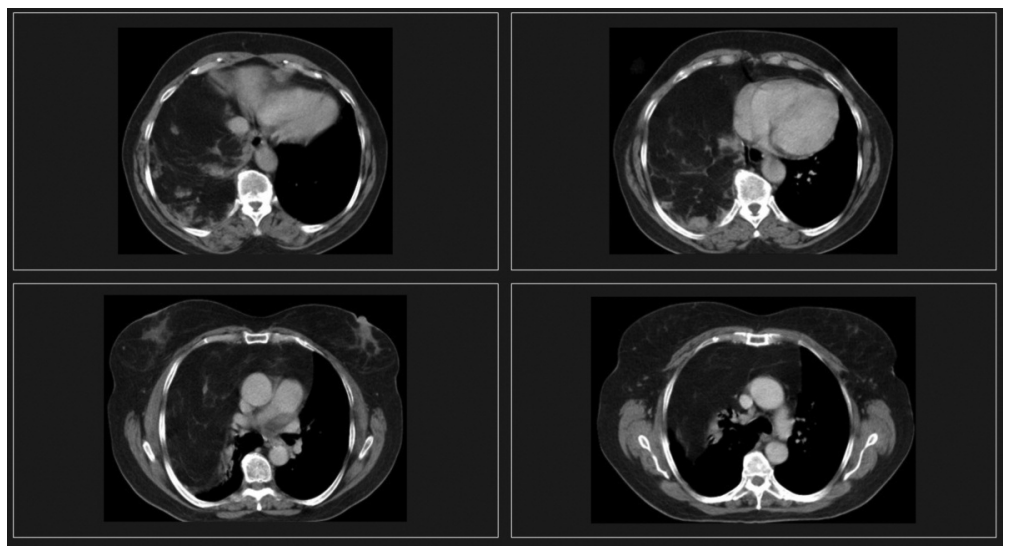

Figure 1. Thorax computer tomography (CT) scan, showing a huge lipomatous tumor occupying anterior mediastinum and the lower right hemithorax.

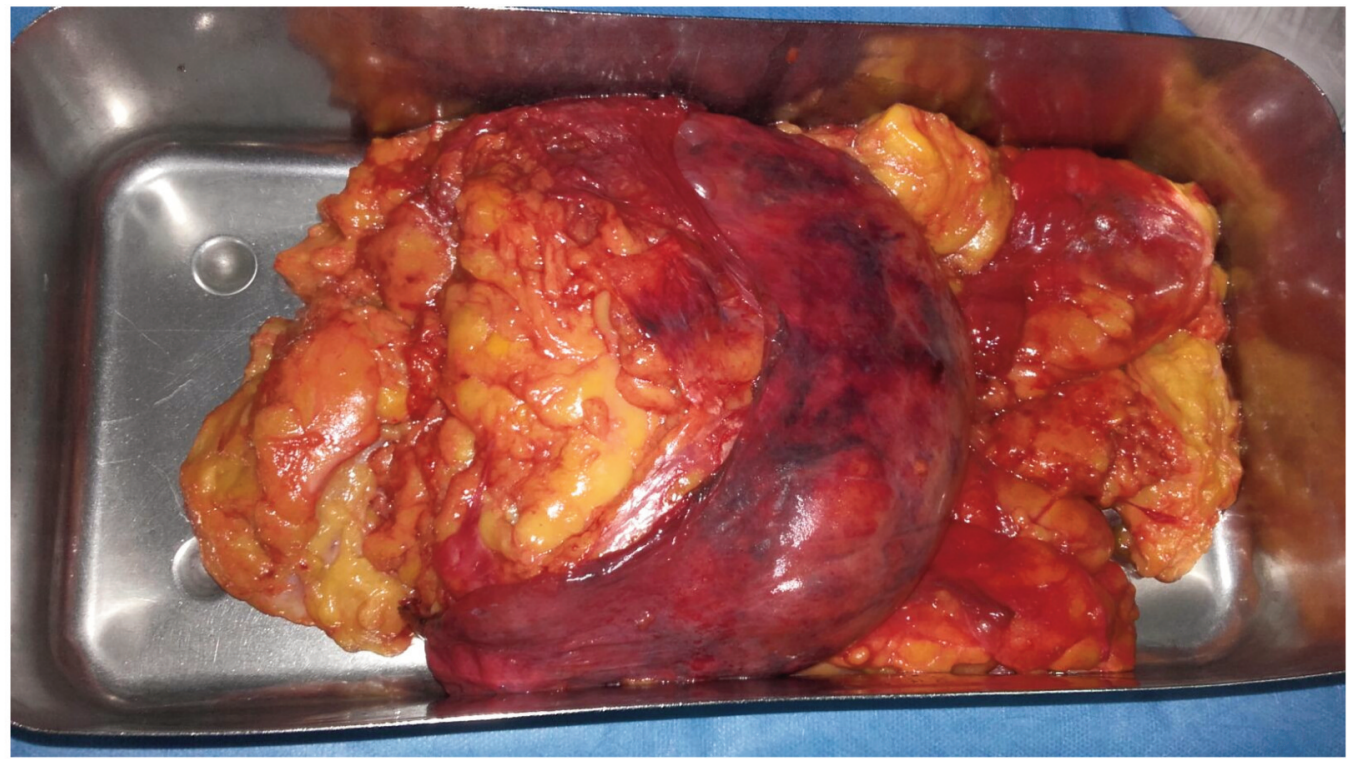

Figure 2. Resection specimen weighting $2.8 \mathrm{~kg}$.

\section{Case Report}

We present the case of a 64-year-old female patient referred to our clinic after a gigantic lipomatous mass was found in her anterior mediastinum and left pleural cavity (Figure 1). The patient came to the clinic with progressive dyspnea that got worse in time and the tumor was found through the imaging examinations performed on this occasion. The patient suspected that the presented progressive symptomatology was due to her weight gain (obese patient, BMI - 41) and ageing and not to the presence of a tumor. The investigations performed in this context revealed no other significant associated comorbidities and the patient was admitted to the surgery department for surgical removal of the intrathoracic mass, assumed to be a gigantic intrathoracic lipoma.

Complete resection of the gigantic intrathoracic lipoma (Figure 2) was performed under general anesthesia, with selective intubation, through a standard right anterolateral thoracotomy. The postoperative course was simple, without complications, the patient was discharged on the fifth postoperative day.

The surprise came with the histopathology results. The macroscopic exam showed that the tumor was gigantic, with a volume of $16 \mathrm{~cm} \times 8 \mathrm{~cm} \times 6 \mathrm{~cm}$, its weight was $2,800 \mathrm{~g}$, well circumscribed, encapsulated in a thin, transparent capsule. The cut surface had a yellow, greasy area which was 


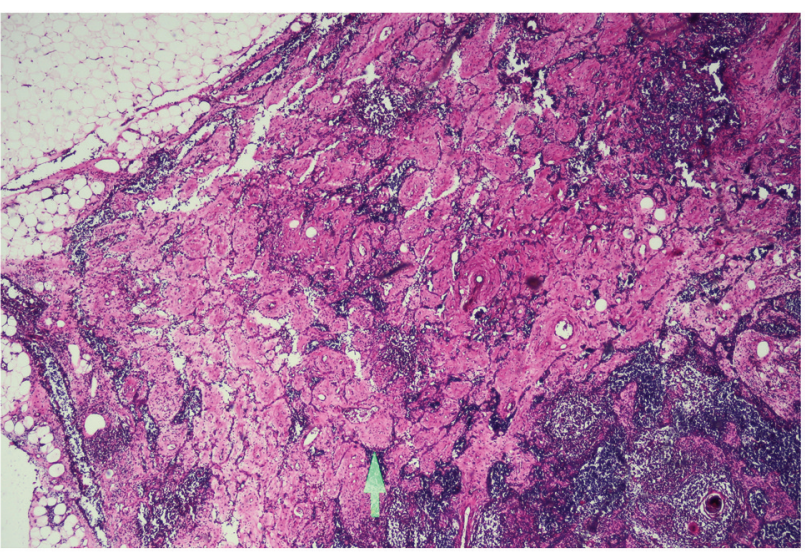

Figure 3. H\&E staining (magnification $\times 4$ ): Lipoma at the left field, fibroadenoma (arrowhead), residual thymus at the bottom right corner.

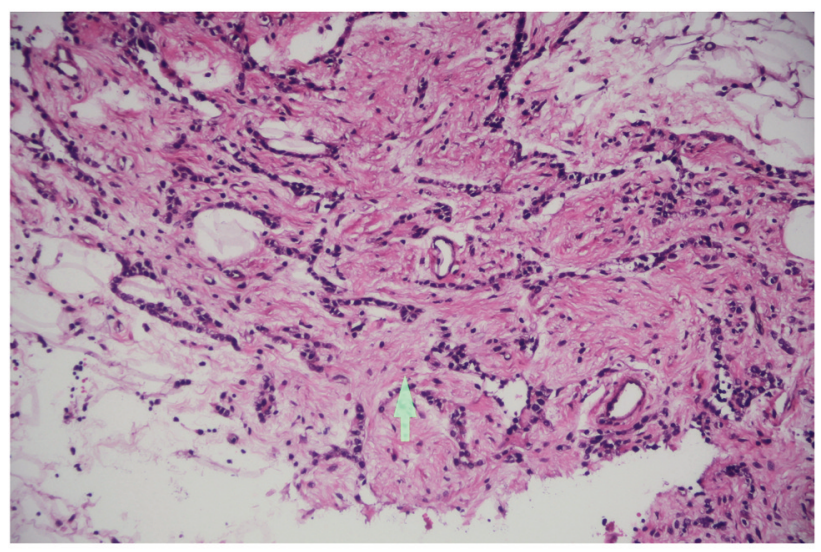

Figure 4. H\&E staining (magnification $\times 10)$ of fibroadenoma: Strands of epithelium (arrowhead) embedded in a dense fibrotic stroma and scattered fat cells.

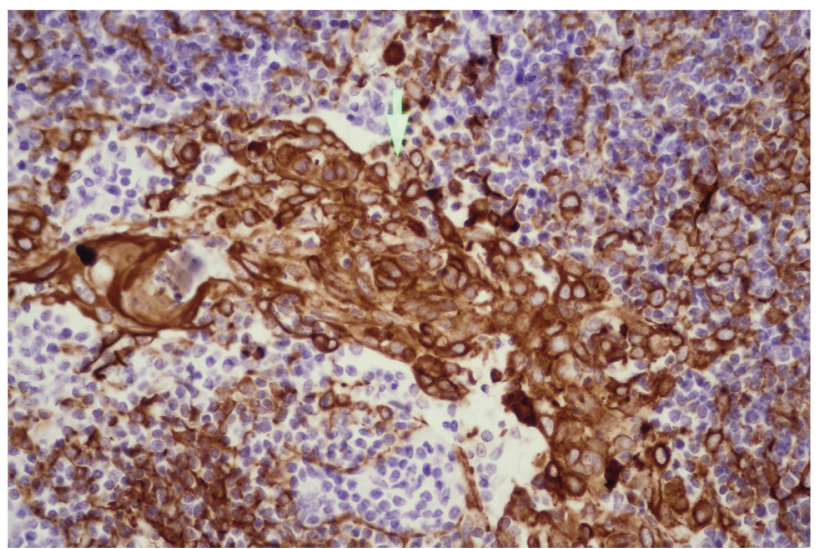

Figure 6. Immunohistochemical studies demonstrating the presence of Cytokeratin AE1/AE3 at the level of epithelial cells (arrowhead), sustaining the diagnosis of lipofibroadenoma.

These aspects (subsequently checked by other anatomopathologists) coincide with the diagnosis of thymic lipofibroadenoma, an extremely rare tumor. On follow-up, four years from surgery, the patient had no particular problems, which was to be expected in the context of a benign tumor.

\section{Discussion}

Lipofibroadenoma is a benign thymic tumor, strongly resembling breast lipofibroadenoma. Etiology is unknown and it is uncertain whether the epithelial or lipofribromatous components (or both) are neoplastic or whether the lesion is a hamartoma.

Only a handful of cases have been reported, all in male patients (3-9). To our knowledge, this case is the first ever 
reported in a woman. Since the number of reported cases is negligible, it is difficult to say whether this is in any way significant.

Complete surgical resection is curative as expected for a benign tumor. Surgical resection can be performed by means of video assisted thoracic surgery (VATS), but is obviously dependent on the tumor's dimensions (8). We are fully aware of the benefits of VATS surgery (10) but in our case the tumor was too large to be removed through a small incision or to allow a good working space within the thorax, so we decided to proceed directly by open surgery. This kind of tumor has a low malignant behavior; there was one case presented with invasion of the mediastinal fat (6), but it is the only one mentioning the possible invasive component.

Associations with B1 type thymoma is described in 2 cases $(3,9)$ so thymic epithelial cell precursor may be responsible for the development of these tumors, but this is only a supposition. Myasthenia gravis, hypogammaglobulinemia and pure red cell aplasia are also described as associated with thymic lipofribroadenoma but it is difficult to say if there is an actual meaning in these pathological associations due to the scarce number of presented cases.

The differential diagnosis is made basically with lipomatous masses within the thorax - thymolipoma (somehow related lesion, but this one has no fibrous component), lipoma, liposarcoma, omental herniation (8). Pathology assessment is the gold standard in making such a diagnosis. Given that these cases are extremely rare, finding them is usually a postoperative surprise.

\section{Conclusion}

Surgeons learn about these kinds of tumors usually after they come across such a case, as they are a postoperative surprise and are extremely rare to encounter. Complete resection is curative and may also be done through minimally invasive surgery, if the circumstances allow it. The number of published cases is minimal and this is probably the first case reported in a woman.

\section{Conflicts of Interest}

The Authors have no conflicts of interest to declare regarding this study.

\section{Authors' Contributions}

$\mathrm{CB}, \mathrm{CP}, \mathrm{IC}, \mathrm{AB}, \mathrm{AH}$ performed the surgical procedure; NB, AA, $\mathrm{MD}, \mathrm{VP}$ reviewed literature data; IrS, IIS, CD, IB preoperative investigation the patient; RS - perioperative and postoperative follow up of the patient, IB, FG FF, DR prepared the draft of the manuscript; IC was advisor of the surgical procedures; EM performed the histopathological and immunohistochemical studies; $\mathrm{CB}, \mathrm{NB}$ reviewed the final version of the manuscript. The Authors read and approved the final version of the manuscript.

\section{References}

1 de Jong WK, Blaauwgeers JL, Schaapveld M, Timens W, Klinkenberg TJ and Groen HJ: Thymic epithelial tumours: a population-based study of the incidence, diagnostic procedures and therapy. Eur J Cancer 44(1): 123-130, 2008. PMID: 18068351. DOI: 10.1016/j.ejca.2007.11.004

2 Engels EA and Pfeiffer RM: Malignant thymoma in the United States: demographic patterns in incidence and associations with subsequent malignancies. Int J Cancer 105(4): 546-551, 2003. PMID: 12712448. DOI: 10.1002/ijc.11099

3 Kuo T and Shih LY: Histologic types of thymoma associated with pure red cell aplasia: a study of five cases including a composite tumor of organoid thymoma associated with an unusual lipofibroadenoma. Int J Surg Pathol 9(1): 29-35, 2001. PMID: 11469342 . DOI: $10.1177 / 106689690100900106$

4 Moran CA, Zeren $\mathrm{H}$ and Koss MN: Thymofibrolipoma. A histologic variant of thymolipoma. Arch Pathol Lab Med 118(3): 281-282, 1994. PMID: 8135632.

5 Qu G, Yu G, Zhang Q, Ma J and Wang X: Lipofibroadenoma of the thymus: a case report. Diagn Pathol 8: 117, 2013. PMID: 23856156. DOI: $10.1186 / 1746-1596-8-117$

6 Aydin Y, Sipal S, Celik M, Araz O, Ulas AB, Alper F and Eroglu A: A rare thymoma type presenting as a giant intrathoracic tumor: lipofibroadenoma. Eurasian J Med 44(3): 176-178, 2012. PMID: 25610236. DOI: 10.5152/eajm.2012.41

7 Onuki T, Iguchi K, Inagaki M and Suzuki K: [Lipofibroadenoma of the thymus]. Kyobu Geka 62(5): 395-398, 2009. PMID: 19425382.

8 Makdisi G, Roden AC and Shen KR: Successful resection of giant mediastinal lipofibroadenoma of the thymus by video-assisted thoracoscopic surgery. Ann Thorac Surg 100(2): 698-700, 2015. PMID: 26234840. DOI: 10.1016/j.athoracsur.2014.09.069

9 Hui M, Paul TR, Uppin SG and Jyothi N: Lipofibroadenoma with B1 thymoma: A case report of a rare thymic tumor. Indian J Pathol Microbiol 61(4): 630-632, 2018. PMID: 30303172. DOI: 10.4103/IJPM.IJPM_443_17

10 Bobocea AC, Trandafir B, Bolca C and Cordoş I: Minimally invasive surgery in cancer. Immunological response. Chirurgia (Bucur) 107(2): 154-157, 2012. PMID: 22712341. 